\title{
Análise Microbiológica de um Centro de Material Esterilizado Hospitalar: Identificação e Resistência a Antibióticos
}

\author{
Bruna Simch ${ }^{1}$, Fabíola Dresch², Mônica Jachetti Maciel ${ }^{3}$
}

\begin{abstract}
RESUMO
Devido à influência da contaminação de superfícies hospitalares na disseminação de microrganismos patogênicos no ambiente hospitalar, este estudo objetivou identificar a microbiota presente em superfícies hospitalares de um Centro de Material Esterilizado (CME) antes e depois da higienização, e avaliar a suscetibilidade das bactérias encontradas diante de antibióticos usados em tratamentos para infecções. As amostras foram coletadas em diferentes superfícies de um CME antes e após a higienização. Os microrganismos foram identificados e após o isolamento, foi realizado o teste de sensibilidade aos antimicrobianos pelo método de disco-difusão conforme padronização do Clinical Laboratory Standards Institute (CLSI). Analisou-se 10 diferentes superfícies em dois momentos, encontrando-se 10 cepas antes da higienização e 8 após $(n=18)$. Acinetobacter baumannii foi o microrganismo mais frequente, representando $50 \%$ das cepas encontradas; seguido de Yersinia pseudotuberculosis, $11,1 \%$, e Staphylococcus coagulase negativa, (SCN) $11,1 \%$. O teste de susceptibilidade a antimicrobianos foi realizado com as 18 cepas e todas apresentaram resistência a, no mínimo, um antibiótico. Diante dos resultados, concluiu-se que $A$. baumannii foi o patógeno encontrado com mais frequência, antes e depois da higienização. E os microrganismos que mais mostraram resistência foram as cepas de A. baumannii, Proteus penneri e SCN, apresentando resistência a quatro antibióticos.

Palavras-chave: Bactérias. Esterilização. Infecção hospitalar. Resistência microbiana.
\end{abstract}

\section{MICROBIOLOGICAL ANALYSIS OF A HOSPITAL STERILIZED MATERIAL CENTER: IDENTIFICATION AND RESISTANCE TO ANTIBIOTICS}

\begin{abstract}
Due to the influence of contamination of hospital surfaces on the dissemination of pathogenic microorganisms in the hospital environment, this study aimed to identify the microbiota present on hospital surfaces of a Sterilized Material Center (CME) before and after the hygiene, and to evaluate the susceptibility of the bacteria found in front to antibiotics used in treatments for infections. Samples were collected on different surfaces of a CME before and after sanitization. The microorganisms were identified and after isolation, an antimicrobial susceptibility test was performed by the disc diffusion method according to standardization of the Clinical Laboratory Standards Institute (CLSI). A total of 10 strains analyzed at each moment, were found 10 strains before hygiene and 8 strains after $(n=18)$. Acinetobacter baumannii was the most frequent microorganism, accounting for $50 \%$ of the strains found; Followed by Yersinia pseudotuberculosis $11.1 \%$ and coagulase negative Staphylococcus (SCN) $11.1 \%$. The antimicrobial susceptibility test was performed with all 18 strains and all showed resistance to at least one antibiotic. Considering the results, it was concluded that $A$. baumannii was the most frequent pathogen, before and after hygiene. The most resistant microorganisms were strains of $A$. baumannii, Proteus penneri and SCN, presenting resistance to four antibiotics.
\end{abstract}

Keywords: Bacteria. Sterilization. Hospital infection. Microbial resistance.

\footnotetext{
Biomédica. Centro de Ciências Biológicas e da Saúde - Universidade do Vale do Taquari - Univates, Lajeado, Rio Grande do Sul, Brasil. brunasimch@gmail.com Biomédica. Especialização em Microbiologia - Faculdade Unyleya. Lajeado, Rio Grande do Sul, Brasil. fdresch@universo.univates.br

${ }^{3}$ Centro de Ciências Biológicas e da Saúde. Programa de Pós-Graduação em Sistemas Ambientais Sustentáveis (PPGSAS) - Universidade do Vale do Taquari Univates. Orcid: http://orcid.org/0000-0002-6863-2181. monicajm@univates.br
} 


\section{INTRODUÇÃO}

O Centro de Material Esterilizado (CME) é uma unidade de apoio técnico dentro do estabelecimento de saúde que tem como principal objetivo fazer com que haja total eliminação de microrganismos oriundos de materiais considerados sujos e contaminados. Os materiais são preparados, descontaminados, esterilizados e armazenados corretamente para distribuição (LEITE, 2008). Conforme a Resolução da Diretoria Colegiada (RDC) no 307/2002 da Agência Nacional de Vigilância Sanitária (Anvisa) (BRASIL 2002), a existência de um CME é obrigatória, sempre que houver centro cirúrgico, centro obstétrico e/ou laboratorial, emergência de alta complexidade e urgência.

Anteriormente à criação dos CMEs, os índices de infecções hospitalares (IHs) eram altíssimos, devido às precárias condições em que as cirurgias e demais procedimentos eram realizadas. Assim sendo, em meados do século 19 tornou-se urgente a criação de um local próprio para preparar e processar os artigos médico-hospitalares utilizados em procedimentos invasivos, a fim de evitar a sua contaminação, bem como reduzir os índices de infecções (LEITE, 2008; COUTO; PEDROSA; NOGUEIRA, 2003; ASCARI, 2012).

Mesmo, entretanto, com os inúmeros avanços na Medicina, atualmente, as IHs ainda representam altos riscos à saúde dos pacientes hospitalizados. Define-se como IHs as infecções contraídas e constatadas após a admissão do paciente, podendo ser manifestadas durante a internação ou após a alta, se relacionadas com a internação ou procedimentos hospitalares. Devido a isso destaca-se a importância do controle da proliferação de microrganismos nos ambientes de saúde, o qual envolve medidas de qualificação da assistência hospitalar em relação à vigilância sanitária, condições de limpeza e desinfecção adequada dos materiais, ambientes e mãos de profissionais da saúde (BRASIL, 1998).

Em relação ao ambiente, incluem-se as superfícies hospitalares, pois embora carreguem um risco mínimo de transmissão direta de infecção, estudos têm demonstrado sua relevante importância em relação à proliferação de microrganismos, pois contribuem para a contaminação cruzada por meio dos profissionais da saúde, atuando, então, como fontes de disseminação de patógenos causadores de infecções relacionadas à saúde. Assim, a higienização correta e eficiente de superfícies e materiais torna-se fundamental no proces- so de prevenção e redução de infecções relacionadas à assistência à saúde (BRASIL, 2012; RUTALA; WEBER, 2004; ASSOCIATION..., 2012).

A desinfecção adequada pode ser ameaçada por vários motivos, entre eles destaca-se a resistência microbiana aos antibióticos e outros agentes antimicrobianos, uma preocupação cada vez maior no âmbito hospitalar. Quando os microrganismos são expostos pela primeira vez a um novo antibiótico, a suscetibilidade tende a ser elevada, assim como a taxa de mortalidade dos microrganismos, fazendo com que poucos sobrevivam dentro de uma população de biIhões de microrganismos, porém os que sobrevivem normalmente apresentam alguma característica genética responsável por sua sobrevivência, tornando-se resistentes a essa e outras drogas (BRASIL, 2007a; TORTORA; FUNKE; CASE, 2012)

$\mathrm{O}$ uso de antimicrobianos em excesso contribui para o surgimento de cepas de microrganismos multirresistentes. A consequência dessa problemática impacta diretamente nas infecções hospitalares, pois conforme os medicamentos são administrados, as cepas dos microrganismos vão adquirindo resistência aos antimicrobianos reduzindo a eficácia do tratamento do paciente, o qual é prejudicado, fazendo com que o tempo de sua internação aumente, aumentando também o risco de contaminação. A racionalização de antimicrobianos oferece a oportunidade de determinar o uso apropriado nos casos em que estão indicados e assim identificar situações nas quais é inadequado o seu uso (BRASIL, 2007a).

A fim de conhecer o perfil microbiológico de superfícies de um $\mathrm{CME}$, o presente estudo tem como objetivos identificar a microbiota presente em superfícies hospitalares antes e depois da higienização, de um Centro de Material Esterilizado (CME) em um hospital do interior do Rio Grande do Sul e avaliar a suscetibilidade das bactérias encontradas diante de antibióticos usados em meio hospitalar.

\section{MATERIAL E MÉTODOS}

Este é um estudo transversal, explicativo e descritivo, com abordagem quantitativa, no qual se buscou a identificação de microrganismos presentes em superfícies de um CME em um hospital de pequeno porte, localizado no interior do Rio Grande do Sul.

O hospital possui 35 leitos, uma sala de cirurgia, um CME, uma sala de recuperação e não possui Unidade de Terapia Intensiva (UTI). O CME está situa- 
do entre a sala de cirurgia e dois banheiros, e não é subdividido em duas partes: a área contaminada e a área limpa. São realizadas semanalmente três a quatro cirurgias, entre quarta e sexta-feira, além de cirurgias de urgência e emergência de baixa complexidade. O hospital não conta com uma Comissão de Controle de Infecção Hospitalar (CCIH).

As coletas foram realizadas em duas etapas: antes de o CME receber o material contaminado, sem higienização prévia do local, e a outra após o material ter passado pelo $\mathrm{CME}$, com a higienização feita por um composto à base de quaternário de amônio. Os pontos de coletas foram: autoclave (coleta interna e externa), superfícies e cubas da pia, válvulas de abertura de torneiras, telefone e frascos de detergente. Nas áreas maiores, tais como superfície da pia, cuba da pia e telefone foram utilizados quadrantes estéreis de tamanho de $5 \mathrm{~cm}$ por $5 \mathrm{~cm}$, totalizando $25 \mathrm{~cm}^{2}$, a fim de delimitar a área a ser pesquisada. As superfícies foram escolhidas por serem áreas onde ocorre maior contato dos profissionais da saúde.

Para a coleta do material foram utilizados swabs previamente umedecidos em um frasco contendo 10 $\mathrm{mL}$ de caldo BHI (Brain Heart Infusion), o quais foram rapidamente pressionados/friccionados sobre a superfície a ser analisada. Logo após a coleta colocou-se os swabs dentro do frasco contendo o caldo BHI, o qual foi transportado em uma caixa térmica até o Laboratório de Microbiologia Didático do Centro Universitário Univates, no qual foram incubados em uma estufa bacteriológica por 24 horas na temperatura de $35 \pm 2$ 으 para serem posteriormente analisados e processados.

$\mathrm{O}$ isolamento e a identificação bacteriana foram realizados de acordo com as metodologias do Manual da Agência Nacional de Vigilância Sanitária (Anvisa) (BRASIL, 2013). Foram utilizados microrganismos como controles negativos e positivos de acordo com o descrito no Manual da Anvisa (BRASIL, 2013). Também se utilizaram controles de esterilidade para os meios de cultura e demais materiais estéreis utilizados. As amostras foram repicadas em ágar sangue de carneiro, e após isoladas repicou-se em ágar TSA (triptona de soja). Após a etapa de isolamento das colônias realizou-se a diferenciação pela coloração de Gram, e seguiu-se o processo de identificação com testes bioquímicos, entre eles o método Bactray ${ }^{\circledR}$ da Laborclin.

Posteriormente à identificação das cepas, foi realizado o teste de sensibilidade aos antimicrobianos pela técnica de disco-difusão em ágar de Kirby-
-Bauer, conforme padronização do Clinical Laboratory Standards Institute (CLSI) (CLINICAL..., 2014). Para os microrganismos Gram-positivos foram utilizados os seguintes antibióticos (Laborclin, Brasil): ampicilina 10 $\mu \mathrm{g}$, clindamicina $2 \mu \mathrm{g}$, gentamicina $10 \mu \mathrm{g}$, levofloxacin $5 \mu \mathrm{g}$, oxacilina $1 \mu \mathrm{g}$ e penicilina $10 \mu \mathrm{g}$. Para os microrganismos Gram-negativos foram utilizados: ampicilina $10 \mu \mathrm{g}$, cefepime $30 \mu \mathrm{g}$, cefuroxima $30 \mu \mathrm{g}$, ceftazidima $30 \mu \mathrm{g}$, gentamicina $10 \mu \mathrm{g}$ e imipenem $10 \mu \mathrm{g}$. A escolha dos antibióticos foi feita conforme o Manual do Antibiograma da Laborclin ${ }^{\circledR}$ (2015) (LABORCLIN, 2013) e também por serem medicamentos utilizados nos tratamentos de infecções hospitalares.

A leitura dos halos encontrados, bem como a interpretação de cepas resistentes, sensíveis e intermediárias foram baseadas nos parâmetros instituídos pelo Manual do Antibiograma da Laborclin ${ }^{\circledR}$ (LABORCLIN, 2013) e pelo BrCAST (Brazilian Committee on Antimicrobial Susceptibility Testing (COMITÊ..., 2005).

Para a expressão de resultados foi realizada análise descritiva dos dados, utilizando números absolutos e dados percentuais dos microrganismos encontrados nas superfícies, bem como dos testes realizados, com auxílio da planilha de cálculo Excel ${ }^{\circledR} 2010$.

\section{RESULTADOS}

Das 10 superfícies amostradas em cada momento, foram encontradas 10 cepas antes da higienização das superfícies e 8 após, num total de 18 cepas $(n=18)$. No total, o microrganismo encontrado com mais frequência foi Acinetobacter baumannii, totalizando $50 \%$ das cepas encontradas $(n=9)$; seguido de Yersinia pseudotuberculosis, $11,10 \%(n=2)$; Staphylococcus coagulase negativa, $11,10 \%(\mathrm{n}=2)$; Providencia rettgeri, 5,56\% ( $n=1)$; Shigella dysenteriae, 5,56\% $(n=1)$; Escherichia fergusonii, 5,56\% ( $n=1)$; Pseudomonas oryzihabitans, $5,56 \%(n=1)$; Proteus penneri $5,56 \%$ $(n=1)$. A frequência dos microrganismos encontrados nas superfícies, é representada na Figura 1.

Antes da higienização houve crescimento microbiano em todas as superfícies, já após a higienização não houve crescimento em duas superfícies apenas, na abertura da torneira de metal e no frasco de detergente. As espécies bacterianas encontradas em cada superfície particularmente estão representadas na Tabela 1. 
Figura 1 - Frequência dos microrganismos encontrados em superfícies do CME de um hospital do interior do Rio Grande do Sul

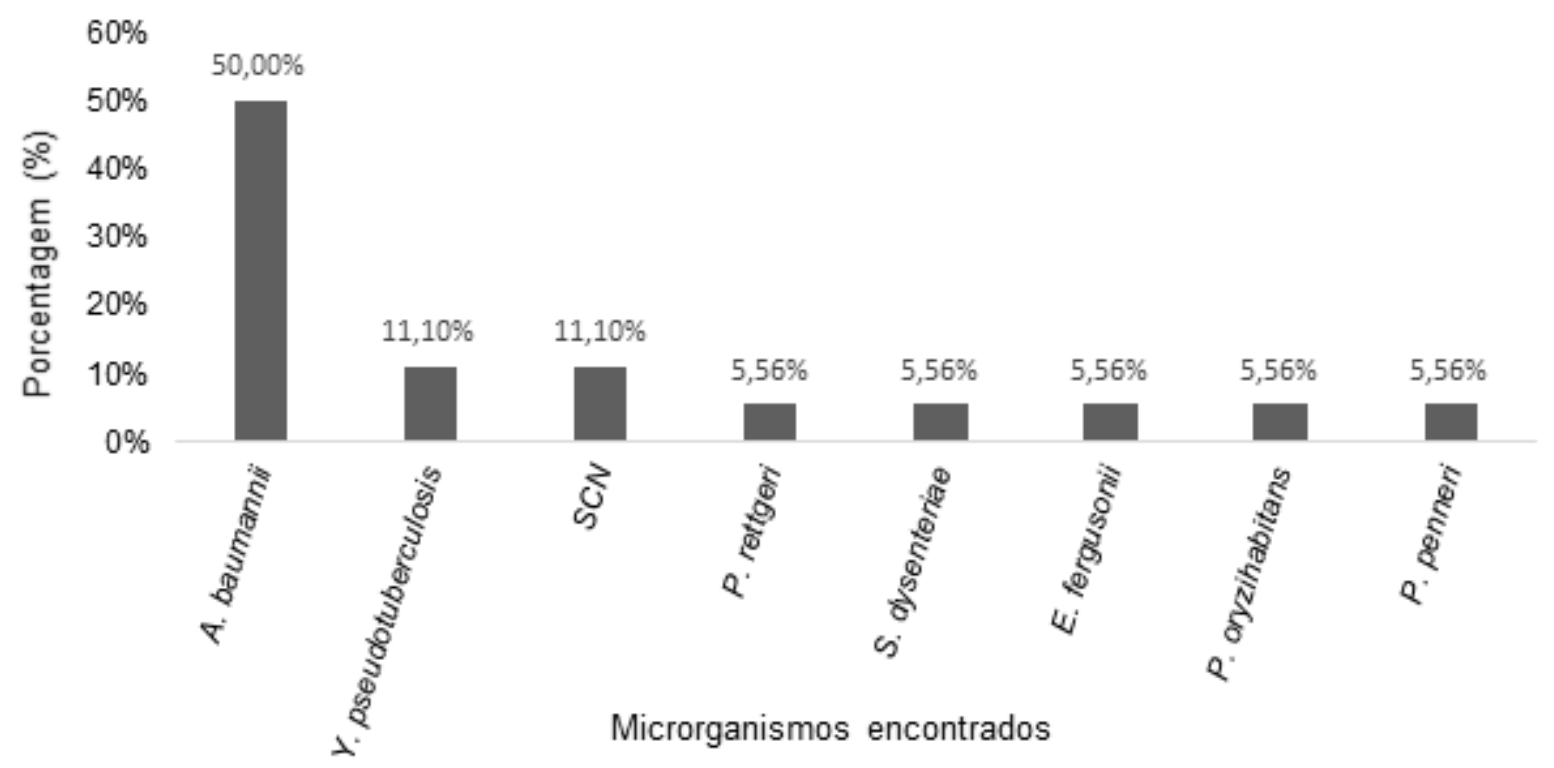

SCN = Staphylococcus coagulase negativa .

Fonte: Dados da pesquisa.

Tabela 1 - Espécies de bactérias encontradas nas superfícies analisadas, antes e depois da higienização, no CME de um hospital do interior do Rio Grande do Sul

\begin{tabular}{|c|c|c|}
\hline SUPERFÍCIE & $\begin{array}{c}\text { ANTES DA HIGIENI- } \\
\text { ZAÇÃO }\end{array}$ & $\begin{array}{c}\text { DEPOIS DA } \\
\text { HIGIENIZAÇÃO }\end{array}$ \\
\hline Autoclave interna & P. rettgeri & S. dysenteriae \\
\hline $\begin{array}{l}\text { Válvula de aber- } \\
\text { tura da autoclave }\end{array}$ & E. fergusonii & P. oryzihabitans \\
\hline $\begin{array}{l}\text { Abertura da tor- } \\
\text { neira de metal }\end{array}$ & Y. pseudotuberculosis & - \\
\hline $\begin{array}{l}\text { Cuba da pia (lado } \\
\text { esquerdo) }\end{array}$ & Y. pseudotuberculosis & A.baumannii \\
\hline Telefone & A. baumannii & A.baumannii \\
\hline $\begin{array}{l}\text { Frasco de deter- } \\
\text { gente }\end{array}$ & A. baumannii & - \\
\hline $\begin{array}{l}\text { Abertura torneira } \\
\text { de plástico }\end{array}$ & A. baumannii & A.baumannii \\
\hline $\begin{array}{l}\text { Superfície da pia } \\
\text { (lado esquerdo) }\end{array}$ & A. baumannii & A.baumanni \\
\hline $\begin{array}{l}\text { Superfície da pia } \\
\text { (lado direito) }\end{array}$ & P. penneri & A.baumannii \\
\hline $\begin{array}{l}\text { Cuba da pia (lado } \\
\text { direito) }\end{array}$ & SCN & SCN \\
\hline
\end{tabular}

- = Sem crescimento bacteriano; SCN = Staphylococcus coagulase negativa.

Fonte: Dados da pesquisa.
O teste de suscetibilidade a antimicrobianos foi realizado com as 18 cepas e todas apresentaram resistência a, no mínimo, um antibiótico. No total, 4 (22\%) apresentaram resistência a 1 antibiótico, 5 (28\%) a 2 antibióticos, 4 (22\%) a 3 antibióticos e 5 (28\%) apresentaram resistência a 4 antibióticos. No total, $78 \%$ dos microrganismos foram resistentes à ampicilina, $\mathrm{e}$ todas as bactérias Gram-negativas apresentaram resistência à cefatzidima.

As cinco cepas que apresentaram maior resistência foram duas cepas de $A$. baumanni, as quais foram encontradas no frasco de detergente (antes da higienização), e no telefone (depois da higienização), uma cepa de $P$. penneri encontrada na superfície da pia do lado direito, outra cepa de A. baumanni localizada na superfície da pia do lado esquerdo (antes da higienização) e uma cepa de SCN encontrada na cuba da pia do lado direito (antes da higienização). As três primeiras cepas citadas foram resistentes à ampicilina, cefepime, ceftazidima e cefuroxima. O terceiro microrganismo citado mostrou-se resistente aos antibióticos ampicilina, ceftazidima, cefuroxima e gentamicina, e por fim o SCN foi resistente à ampicilina, clindamicina, oxacilina e penicilina. 
Tabela 2 - Porcentagem da resistência aos antibióticos apresentada pelas bactérias Gram negativas encontradas nas superfícies analisadas de um hospital do interior do Rio Grande do Sul

\begin{tabular}{|c|c|c|c|c|c|c|}
\hline BACTÉRIAS & & IBIÓTIC & RESISTÊN & (\%) & & \\
\hline ANTIBIÓTICOS & AMP & CPM & CAZ & CRX & GEN & IPM \\
\hline A. baumanni $(\mathrm{n}=9)$ & $89 \%$ & $22 \%$ & $100 \%$ & $67 \%$ & $11 \%$ & $0 \%$ \\
\hline Y. pseudotuberculosis $(\mathrm{n}=2)$ & $50 \%$ & $0 \%$ & $100 \%$ & $0 \%$ & $0 \%$ & $0 \%$ \\
\hline P. rettgeri $(n=1)$ & $0 \%$ & $0 \%$ & $100 \%$ & $0 \%$ & $0 \%$ & $0 \%$ \\
\hline S. dysenteriae $(n=1)$ & $0 \%$ & $0 \%$ & $100 \%$ & $0 \%$ & $0 \%$ & $0 \%$ \\
\hline E. fergusonii $(\mathrm{n}=1)$ & $0 \%$ & $0 \%$ & $100 \%$ & $0 \%$ & $0 \%$ & $0 \%$ \\
\hline P. penneri $(n=1)$ & $100 \%$ & $100 \%$ & $100 \%$ & $100 \%$ & $0 \%$ & $0 \%$ \\
\hline P. oryzihabitans $(n=1)$ & $100 \%$ & $0 \%$ & $100 \%$ & $100 \%$ & $0 \%$ & $0 \%$ \\
\hline
\end{tabular}

$\mathrm{AMP}=$ ampicilina; $\mathrm{CPM}$ = cefepime; $\mathrm{CAZ}$ = ceftazidima; $\mathrm{CRX}=$ cefuroxima; $\mathrm{GEN}$ = gentamicina; $\mathrm{IPM}$ = imipenem.

Fonte: Dados da pesquisa.

A suscetibilidade das cepas Gram negativas apresentadas aos antibióticos testados pode ser vista na Tabela 2. A resistência expressa em porcentagem (\%) foi calculada com todas as cepas de cada espécie de microrganismo encontrado.

As duas cepas de Staphylococcus coagulase negativa encontradas demonstraram resistência não só à oxacilina, mas também à penicilina, simultaneamente, podendo-se caracterizá-las como Staphylococcus meticilina resistente (MRS) (CLINICAL..., 2014; LABORCLIN, 2013).

\section{DISCUSSÃO}

No presente estudo $50 \%$ dos microrganismos encontrados foram identificados como Acinetobacter baumannii. Este gênero é detectado principalmente em pacientes e ambientes de alto risco, como em UTIs, e mostra-se responsável por altos índices de gravidade e mortalidade (URBAN; SEGAL-MAURER; RAHAL, 2003; WEBER et al., 2010). É um agente responsável por quase todas infecções adquiridas em UTI, particularmente infecções do trato respiratório (CLINICAL..., 2014).

A. baumannii é um microrganismo encontrado com mais frequência em hospitais, já relatados em pacientes, em circuito respiratório, torneiras, pias, ressuscitadores, roupas de cama, superfícies fixas e objetos inanimados, contudo já foram isolados também em alimentos congelados, dieta enteral, esgotos e no ar (BRASIL, 2007a; ZANON; NEVES, 1987; CISNEIROS et al., 1996). No presente estudo $A$. baumannii foi encontrado na superfície da pia, tanto do lado esquerdo como do lado direito, assim como na cuba, na torneira de plástico e no frasco de detergente, desenvolvendo-se em superfícies de metal e plástico. $A$. baumannii é um patógeno capaz de sobreviver em superfícies inanimadas por um longo período (URBAN; SEGAL-MAURER; RAHAL, 2003; WEBER et al., 2010).

Cepas de Acinetobacter baumannii apresentam, na maioria das vezes, multirresistência e possuem capacidade de sobreviver por muito tempo em diversas superfícies, incluindo aço inoxidável, cerâmica, cloreto de polivinil (PVC) e borracha (WEBER et al., 2010). No estudo de Wendt et al. (1997) foi avaliada in vitro a capacidade de sobrevivência de cepas de $A$. baumannii. Foram testadas, no total, 10 estirpes em quatro superfícies, com uma taxa de $50 \%$ de sobrevivência em até 16 semanas. Wendt et al. concluíram que a umidade relativa influencia na sobrevivência da espécie analisada, contudo as estirpes de $A$. baumannii isoladas de lugares não úmidos exibiram sobrevivência prolongada em superfícies secas quando comparadas com superfícies úmidas, mostrando, então, que a fonte de isolamento e demais características da cepa podem influenciar no seu tempo de vida (WENDT et al., 1997). Essa informação é importante, pois em casos de surtos, pode-se se esperar que as fontes das cepas sejam locais mais secos, já indicando uma desinfecção extensiva dos possíveis ambientes.

A capacidade que as estirpes de $A$. baumannii têm de sobreviver por longos período em superfícies influencia diretamente no processo desinfecção, o qual é prejudicado, e consequentemente no aumento do risco de transmissão cruzada do microrganismo (WEBER et al., 2010; WENDT et al., 1997). No presente estudo tal patógeno foi encontrado em superfícies 
que pertencem a uma mesma área (pia, torneira e frasco de detergente), além de serem locais de intenso contato de profissionais da saúde, que constantemente higienizam-se antes de entrar na sala de cirurgia, podendo assim ocasionar uma possível disseminação de microrganismos.

Outro microrganismo de importante relevância em infecções hospitalares, principalmente em Infecções de Sítio Cirúrgico (ISC), já relatados em amostras de ar e mesas de salas cirúrgicas, é o Staphylococcus spp. (AMARAL et al., 2013; ALEXANDER et al., 2013; MANGRAM et al., 1999). Neste estudo foram encontradas, mais especificamente, cepas da espécie SCN em amostras de superfícies de cubas de pias. Ainda que SCN não apresente relevância clínica em infecções comunitárias, uma vez que fazem parte da microbiota humana, no ambiente hospitalar representa o segundo agente causador de ISC, antecedido apenas por Staphylococcus aureus (ALEXANDER et al., 2013; CATALANO et al., 1999). Bactérias como SCN tornam-se patogênicas quando agem como oportunistas causadoras de infecções, levando em conta a carga microbiana, além da imunidade do paciente e os tratamentos e procedimentos aos quais ele é exposto (BRASIL, 2013; BARRETO; PICOLI, 2008).

No estudo de Alexander et al. (2013) foi avaliada a contaminação de possíveis sítios de infecção em salas cirúrgicas, salientando a importância das superfícies como reservatório de microrganismos, pois observaram que superfícies mais utilizadas diariamente pelos profissionais da saúde, como telefones, mouses, mesas, apresentaram maior contaminação bacteriana quando comparadas àquelas que não são usadas frequentemente, mostrando a importância da higienização/desinfecção adequada das mãos dos profissionais da saúde, bem como da manutenção do ambiente esterilizado.

A existência de estirpes de SCN resistentes no ambiente hospitalar, inclusive em salas cirúrgicas, tem aumentado cada vez mais, e já vem sendo citada por diversos autores, principalmente a resistência à oxacilina, que é uma das primeiras escolhas de antibiótico utilizados para infecções de Staphylococcus spp. já resistentes (ALEXANDER et al., 2013; MANGRAM et al., 1999; BOYCE et al., 1997). Tal fato corrobora com este estudo, no qual as cepas de SCN encontradas também apresentaram resistência à oxacilina, podendo, ainda, serem caracterizadas como MRS, dado que se mostraram resistentes também à penicilina.

Um outro patógeno que raramente causa infecções humanas, mas por acometer hospedeiros imunocomprometidos tem sido considerado potencialmen- te nosocomial, sendo encontrado, neste estudo, na válvula de autoclave, é a Pseudomonas oryzihabitans (LAM et al., 1994; LIN et al., 1997; DECKER; SIMON; KEISER, 1991). No ambiente hospitalar, $P$. oryzihabitans tem sido recuperada em pias, drenos, apareIhos de terapia e assistência respiratória (CHAUDHRY; SCHOCH; CUNHA, 1992, STEINBERG; RIO, 2005), até mesmo em gaze salina, como reportado no estudo de Woo et al (2014). Tais fatos tornam-se perigosos ao paciente, visto que podem colonizar materiais que são utilizados diretamente em seus procedimentos.

Em relação às cepas de Proteus penneri encontradas no estudo, tal fato pode estar relacionado com a higienização de frascos de urinas e materiais de sondagem na pia do CME, uma vez que esse microrganismo é isolado principalmente a partir da urina (50\%), exsudatos de feridas e tecidos moles (25\%), e culturas de sangue (15\%), principalmente de origem nosocomial (CANTÓN; SÁNCHEZ-MORENO; MOROSINI REILLY, 2006). Entre as cirurgias realizadas na instituição pesquisada está a cesariana, na qual é colocada uma sonda urinária na paciente para a realização do procedimento.

A resistência à ampicilina e cefuroxima da cepa de $P$. penneri encontrada neste estudo, vai ao encontro dos resultados presentes no estudo de Kishore (2012), no qual todos os isolados apresentaram resistência à ampicilina, e outros, ainda, à cefalosporinas (cefalexina e cefuroxima) também.

Já em referência às cepas de Providencia rettgeri não foram encontradas relações específicas entre o centro cirúrgico e CME. Em um estudo realizado no Japão, no entanto, foram identificadas 9 cepas deste microrganismo em um total de 495 casos clínicos de dois hospitais, das quais 6 cepas do mesmo hospital foram encontradas em amostras de urina de pacientes diferentes, e outras 2 cepas foram isoladas a partir de amostras de escarros e sangue de pacientes (SHIROTO et al., 2005). Já no estudo de Yoh et al (2005), espécies de Providencia spp. foram relatadas como causas de diarreia de viajantes. Em um total de 130 isolados, 9 foram identificados como Providencia rettgeri, nos quais os pacientes relatavam queixa de dores abdominais e vômitos. Logo, os resultados dos estudos mostraram que espécies de Providencia spp. podem ser consideradas importantes agentes patogênicos (SHIROTO et al., 2005; YOH et al., 2005).

Assim como as espécies de Providencia spp., bactérias do gênero Shigella apresentam sintomas e patogenicidade bastante semelhantes, e também não foram encontrados na literatura estudos que comprovem relações da identificação desse patógeno em 
CME. É válido ressaltar, porém, que algumas estirpes de Shigella dysenteriae possuem capacidade de produzir exotoxinas, causando, assim, disenterias graves. São encontradas, com mais frequência, em amostras de fezes, como no estudo realizado no Laboratory of Children Medical Center Hospital (POURAKBARI et al., 2010).

Do gênero Escherichia, no presente estudo, foi encontrada apenas uma cepa de Escherichia fergusonii, a qual, de acordo com a literatura, não tem sido relatada em pesquisas envolvendo CME. Sabe-se, contudo, que bactérias deste gênero fazem parte da microflora normal do intestino, no entanto algumas estirpes podem ser patogênicas e capazes de induzir doenças intra ou extraintestinais, apresentando riscos significativos à saúde. É possível, portanto, relacionar a presença de Escherichia fergusonii com contaminação cruzada, uma vez que o gênero Escherichia está relacionado com infecções de cirurgias abdominais (FORGETTA et al., 2012; BREATHNACH, 2013) e o hospital do presente estudo realiza cirurgias abdominais, como abdominoplastia e lipoaspiração.

Assim sendo, diante dos resultados apresentado neste estudo e visto que os locais que apresentaram contaminação eram superfícies de intenso contato por parte da equipe de saúde, é possível justificar a presença dos microrganismos encontrados, devido, principalmente, $\grave{a}$ transmissão de contaminação por parte das mãos de profissionais da saúde, ou até mesmo materiais e amostras contaminados, podendo ser considerados como veículos de contaminação (BRASIL, 2012; RUTALA; WEBER, 2004). Caso a higienização não seja feita de maneira correta, o fato de muitos profissionais trabalharem em outros hospitais, tendo contato com cepas, como também com pacientes contaminados vindos de outras unidades de saúde, pode influenciar na transmissão de microrganismos (WEBER et al., 2010). Além disso, o CME encontra-se entre o Centro Cirúrgico e dois banheiros e, na sequência, há uma Sala de Recuperação. Toda a ala que abrange essas quatro áreas possui apenas uma porta, o que também pode ser relacionado com contaminação de superfícies e proliferação de patógenos.

\section{CONCLUSÕES}

As superfícies analisadas no presente estudo apresentaram crescimento microbiano, tanto antes quanto depois da higienização. No total de microrganismos identificados, Acinetobacter baumannii foi isolado com maior frequência, representando $50 \%$ dos microrganismos encontrados, seguido de Staphylococcus coagulase negativa ( $\mathrm{SCN}$ ) e Yersinia pseudotuberculosis, ambas representando $11 \%$.

Em relação à atividade antimicrobiana analisada no presente estudo, as cepas de $A$. baumannii, Proteus penneri e SCN apresentaram maior perfil de resistência, mostrando-se resistentes a quatro antibióticos.

Nesse contexto, tendo em vista a capacidade que algumas bactérias, como Acinetobacter spp., têm de sobreviverem por muito tempo em superfícies, a presença de enterobactérias antes, e principalmente depois da higienização, indica a necessidade de um maior cuidado e atenção em relação à desinfecção correta das superfícies analisadas. $O$ desinfetante químico deve ser analisado, bem como o seu tempo de contato e concentração de uso. Além disso, em ambientes como CMEs são manipulados muitos materiais que, posteriormente, entrarão em contato diretamente com o paciente, por isso destaca-se também a importância da conscientização em relação à higienização das mãos por parte dos profissionais da saúde, para dessa forma minimizar os riscos de contaminação cruzada entre pacientes, profissionais da saúde e ambientes hospitalares, e evitar possíveis casos de infecções hospitalares.

\section{REFERÊNCIAS}

ASSOCIATION FOR THE ADVANCEMENT OF MEDICAL INSTRUMENTATION (AAMI). Comprehensive guide to steam sterilization and sterility assurance in health care facilities - ANSI/AAMI ST79. 2010, A3. 2012.

ALEXANDER, J. W. et al. Surveillance of bacterial colonization in operating rooms. Surgical infections. v. 14, p. 345351, 2013.

AMARAL, A. M. et al. Importância da proteção da mesa de instrumentais cirúrgicos na contaminação intraoperatória de cirurgias limpas. Rev. Latino-Am. Enfermagem. v. 21, n. 1, p. 426-432, 2013.

ASCARI, R. A. et al. Fluxo de materiais odonto-médico-hospitalares durante o seu processamento em unidades básicas de saúde. Rev. Udesc em Ação, v. 6, n. 1, p. 1-16, 2012.

BARRETO, M. F.; PICOLI, S.U. Staphylococcus in a hospital in Porto Alegre (RS). Rev. Bras. de Análises Clínicas, v. 40, p. 285-287, 2008.

BOYCE, J. M. et al. Environmental contamination due to methicillin-resistant Staphylococcus aureus: possible infection control implications. Infect Control Hosp Epidemiol., v. 18, n. 9, p. 622-627, 1997. 
BRASIL. Agência Nacional de Vigilância Sanitária (Anvisa). Ministério da Saúde (MS). Investigação e controle de bactérias multirresistentes. 2007a. Disponível em: <http://www. anvisa.gov.br/servicosaude/controle/reniss/manual\%20_ controle_bacterias.pdf>. Acesso em: 18 set. 2016.

. Agência Nacional de Vigilância Sanitária (Anvisa). Ministério da Saúde (MS). Microbiologia clínica para o controle de infecção relacionada à assistência à saúde. Módulo 6: Detecção e identificação de bactérias de importância médica. 1. ed. 2013. Disponível em: <http://www20.anvisa. gov.br/segurancadopaciente/index.php/publicacoes/item/ deteccao-e-identificacao-de-bacterias-de-importancia-medica>. Acesso em: 19 set. 2016.

Agência Nacional de Vigilância Sanitária (Anvisa). Ministério da Saúde (MS). Manual de controle de bactérias. 2007b. Disponível em: <http://www.anvisa.gov.br/servicosaude/controle/reniss/manual\%20_controle_bacterias. pdf>. Acesso em: 19 set. 2016.

. Ministério da Saúde. Portaria $n^{\circ} 2.616$, de 12 de maio de 1998 . Dispõe sobre a obrigatoriedade da manutenção pelos hospitais do país, de programa de controle de infecções hospitalares. Ministério da Saúde, 1998. Disponível em: <http://www.saude.mg.gov.br/images/documentos/ portaria_2616.pdf>. Acesso em: 19 set. 2016.

. Ministério da Saúde. Resolução (RDC) no 15, de 15 de março de 2012. Dispõe sobre requisitos de boas práticas para o processamento de produtos para saúde e de outras providências. Ministério da Saúde, 2012. Disponível em: <http://www20.anvisa.gov.br/segurancadopaciente/index. php/legislacao/item/rdc-15-de-15-de-marco-de-2012>. Acesso em: 18 set. 2016.

. Ministério da Saúde. Resolução (RDC) no 307, 14 de novembro de 2002. Dispõe sobre o Regulamento Técnico para planejamento, programação, elaboração e avaliação de projetos físicos de estabelecimentos assistenciais de saúde. Ministério da Saúde, 2002. Disponível em: <http://portal. anvisa.gov.br/wps/wcm/connect/3f54b800474597439fb7df3fbc4c6735/RDC+N\%C2\%BA+307-2002.pdf?MOD=AJPERES>. Acesso em: 18 set. 2016.

BREATHNACH, A. S. Nosocomial infections and infection control. Medicine. v. 41, p. 649-653, 2013.

CANTÓN, R.; SÁNCHEZ-MORENO, M. P.; MOROSINI REILLY, M. I. Proteus penneri. Enferm Infecc Microbiol Clin., v. 1, p. 8-13, 2006.

CATALANO, M. et al. Survival of Acinetobacter baumannii on bed rails during an outbreak and during sporadic cases. $J$ Hosp Infect., v. 42, p. 27-35, 1999.

CENTRO DE VIGILÂNCIA EPIDEMIOLÓGICA (CVE). Secretaria de Estado da Saúde de São Paulo. Vigilância epidemiológica das doenças transmitidas por alimentos: investigação de surtos. São Paulo, 2003. Disponível em: <ftp://ftp.cve. saude.sp.gov.br/doc_tec/hidrica/yersi_entero.pdf>. Acesso em: 19 set. 2016.

CHAUDHRY, H. J.; SCHOCH, P. E.; CUNHA, B. A. Flavimonas oryzihabitans (CDC group Ve2). Infect Control Hosp Epidemiol. v. 13, p. 485-48, 1992.
CISNEIROS, J. M. et al. Bacteremia due to Acinetobacter baumannii: epidemiology, clinical findings, and prognostic features. Journal Clinical Infectious Diseases, v. 22, n. 6, p. 1.026-1.032, 1996.

CLINICAL AND LABORATORY STANDARDS INSTITUTE (CLSI). Performance Standards for Antimicrobial Susceptibility Testing: Twenty-Fourth Informational Supplement. M100 S-24, v. 34, n. 1, 2014.

COMITÊ BRASILEIRO DE TESTES DE SENSIBILIDADE AOS ANTIMICROBIANOS (BrCast). Método de disco Fusão. Versão 5.0. 2005. Disponível em: <http://brcast.org.br/documentos/>. Acesso em: 21 set. 2016.

COUTO, R. C.; PEDROSA, T. M. G.; NOGUEIRA, J. M. Infecção hospitalar e outras complicações não-infecciosas da doença: epidemiologia, controle e tratamento. 3. ed. Rio de Janeiro: Medsi. 2003.

DECKER, C. F.; SIMON, G. L.; KEISER, J. F. Flavimonas oryzihabitans (Pseudomonas oryzihabitans; CDC group Ve-2) bacteremia in the immunocompromised host. Arch Intern Med., v. 151, p. 603-604, 1991.

FORGETTA, V. et al. Pathogenic and multidrug-resistant Escherichia fergusonii from broiler chicken. Poultry Science, v. 91, n. 2, p. 512-525, 2012.

KISHORE, J. Isolation, identification \& characterization of Proteus penneri - a missed rarepathogen. Indian J Med. Res., v. 135, n. 3, p. 341-345, 2012.

LABORCLIN. Manual do antibiograma. 2013. 32p. Disponível em: <http://www.interlabdist.com.br/dados/noticias/ pdf_238.pdf>. Acesso em: 21 set. 2016.

LAM, S. et al. Community-acquired soft-tissue infections caused by Flavimonas oryzihabitans. Clin Infect Dis. v. 18, p. 808-809, 1994.

LEITE, F. B. Central de material esterilizado: projeto de reestruturação e ampliação do Hospital Regional de Francisco Sá. Centro Universitário Euroamericano-Unieuro. 2008. Disponível em: <http://bvsms.saude.gov.br/bvs/artigos/artigo_CME_flavia_leite.pdf>. Acesso em: 18 set. 2016.

LIN, R. D. et al. Flavimonas oryzihabitans bacteremia: clinical features andmicrobiological characteristics of isolates. Clin Infect Dis., v. 24, p. 867-873, 1997.

MANGRAM, A. J. et al. Guideline for prevention of surgical site infection. Infection control and hospital epidemiology., v. 20, p. 247-278, 1999.

POURAKBARI, B. et al. Frequency and antimicrobial susceptibility of Shigella species isolated in Children Medical Center Hospital, Tehran, Iran, 2001-2006. Braz J Infect Dis., v. 14, n. 2, p. 153-157, 2010.

RUTALA, W. A.; WEBER, D. J. The benefits of surface disinfection. Ame. Journal Infec. Control., v. 32, p. 226-231, 2004.

SANTOS, N. Q. A resistência bacteriana no contexto da infecção hospitalar. Texto Contexto Enfermagem, v. 13, p. 6470, 2004.

SHIROTO, K. et al. Metallo- $\beta$-lactamase IMP-1 in Providencia rettgeri from two different hospitals in Japan. Journal Medical Microbiol., v. 54, n. 11, p. 1.065-1.070, 2005. 
STEINBERG, J. P.; RIO, C. D. Other gram-negative and gram-variable bacilli. In: MANDELL, G. L.; BENNETT, J. E.; DOLIN, R. Principles and Practices of Infectious Diseases. 6. ed. Nova York: Churchill Livingstone, 2005.

TORTORA, G. J.; FUNKE, B. R.; CASE, C. L. Microbiologia. 10. ed. Porto Alegre: Artmed, 2012.

URBAN, C.; SEGAL-MAURER, S.; RAHAL, J. Considerations in control and treatment of nosocomial infections due to multidrug-resistant Acinetobacter baumannii. Clinical Infectious Diseases, v. 36, p. 1.268-1.274, 2003.

WEBER, D. J. et al. Role of hospital surfaces in the transmission of emerging health care-associated pathogens: norovirus, Clostridium difficile, and Acinetobacter species. American Journal of Infec. Control., v. 38, n. 5, p. 25-33, 2010.
WENDT, C. et al. Survival of Acinetobacter baumannii on dry surfaces. J Clin Microbiol., v. 35, p. 1.394-1.397, 1997.

WOO; K. S. et al. Outbreak of Pseudomonas Oryzihabitans Pseudobacteremia. Infection e Chemotherapy, v. 46, n. 1, p. 42-44, 2014.

$\mathrm{YOH}, \mathrm{M}$. et al. Importance of Providencia species as a major cause of travellers' diarrhea. Journal Med. Microbiol., v. 54, n. 11, p. 1.077-1.082, 2005.

ZANON, U.; NEVES, J. Infecções hospitalares: prevenção, diagnóstico, tratamento. Rio de Janeiro: Medsi, 1987. 